\title{
Uso de redes sociales por estudiantes de pregrado de una facultad de medicina en Lima, Perú
}

\author{
Use of social networks by pre-graduate students in a school of medicine in Lima, Peru \\ Otto Barnaby Guillén López ${ }^{1, a, b}$ \\ RESUMEN
}

El uso de internet ha aumentado en la última década. Los estudiantes universitarios utilizan el internet con las redes sociales, no solo en actividades de recreación sino también durante sus actividades educativas. Incluso, algunas facultades de medicina las usan como recurso pedagógico en algunos cursos. Objetivo: Describir algunas formas de uso de algunas redes sociales por los estudiantes de pregrado de una facultad de medicina. Material y métodos: Estudio descriptivo transversal. Se realizó una encuesta a alumnos del quinto año de una Facultad de Medicina de Lima, Perú. Se preguntó sobre las redes sociales en las que tenían cuenta, frecuencia de uso, y tiempo que pasan en Facebook ${ }^{\circledR}$ y YouTube $\AA$. Resultados: Respondieron 52 alumnos. Todos tenían cuenta en Facebook ${ }^{\circledR}, 46,2 \%$ en Instagram ${ }^{\circledR}, 34,6 \%$ en Twitter ${ }^{\circledR}, 15,4 \%$ en SlideShare ${ }^{\circledR}$ y 7,7\% en LinkedIn ${ }^{\circledR}$. De los que tenían Facebook ${ }^{\circledR}$, $50 \%$ lo utilizaban una hora o más diariamente. El 63,5\% visualizaban videos en YouTube ${ }^{\circledR}$ una o más veces al día. Conclusiones: Se encontró que todos los estudiantes tienen al menos una cuenta en redes sociales, de preferencia en Facebook ${ }^{\circledR}$, y lo usan al menos una vez al día, similar a lo encontrado en otros países.

PALABRAS CLAVE: Redes sociales, aprendizaje, educación, enseñanza. (Fuente: DeCS BIREME).

\section{SUMMARY}

The use of the internet has increased significantly over the last decade. Pre-graduate students use the internet and social networks not only for recreational activities but also for academic activities. Moreover, some schools of medicine use these resources to teach medical students. Objective: To describe the use of social networks by pregraduate medical students. Methods: A cross-sectional study was carried-out by using a questionnaire among pregraduate medical students of the fifth year. Wes asked about which social network the student has an active account,

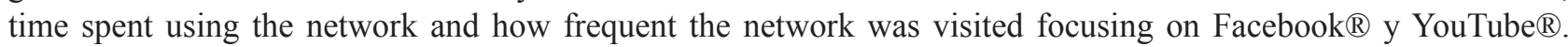
Results: 52 students participated; all of them had an account in Facebook ${ }^{\circledR}, 46.2 \%$ in Instagram ${ }^{\circledR}, 34.6 \%$ in Twitter $\AA$, $15.4 \%$ in SlideShare ${ }^{\circ}$ y $7.7 \%$ in LinkedIn $₫$. Among those who had an account in Facebook ${ }^{\circledR}, 50 \%$ use it at least one-hour per day; $65.3 \%$ watched videos in YouTube ${ }^{\circledR}$. Conclusions: All students used at least one social network, mostly Facebook ${ }^{\circledR}$, and use it at least once per day, this finding is similar to what has been reported elsewhere.

KEYWORDS: Social Networking, learning, education, teaching. (Source: MeSH NLM).

\footnotetext{
Facultad de Medicina. Universidad Peruana Cayetano Heredia. Lima, Perú.

Profesor Auxiliar.

Médico Internista.
} 


\section{INTRODUCCIÓN}

En Lima Metropolitana, Perú, el uso de internet de al menos una vez al día se incrementó de $29 \%$ a $65 \%$ en la población de 17 a 24 años de edad entre el año 2009 al 2016. En el 2016, el 45\% de la población usa el internet para buscar información, comunicarse y entretenerse (1). Entre los usos del internet se encuentran las redes sociales, que en la actualidad son utilizadas por millones de personas, incluyendo a $72 \%$ de la generación "Z" o "nativos digitales" que reportan su uso, lo que ha hecho que el lugar, la forma y el momento de comunicarse con y entre ellos sean diferentes en esta época (2).

El diccionario de la Real Academia Española (RAE) define "Red Social" como "una plataforma digital de comunicación global que pone en contacto a gran número de usuarios" (3) y el Diccionario Cambridge (DC) define "Social Network" como "un sitio web o programa de computador que permite a las personas comunicarse y compartir información en el internet usando una computadora o teléfono celular o móvil"(4). Aunque las redes sociales más conocidas son Facebook, Google+, Instagram, LinkedIn, Pinterest, Reddit, Twitter, YouTube, entre otros (5), de acuerdo a las definiciones de la RAE y DC, se puede considerar a algunos repositorios como SlideShare como una red social, incluso el Institute of Education de la University College London (UCL) la considera una "red social para investigadores" (6).

Si bien, la mayor parte del uso de dichas redes sociales son para actividades de recreación (chatear, socializar, "postear", jugar online), muchas personas también las usan para aprender como puente entre el aprendizaje formal e informal, llegando a promoverse como parte de una teoría de aprendizaje conectivista (7).

En el Penn State College of Medicine están usando las redes sociales como recurso educativo en un curso de humanidades, reportando beneficios como: escribir sucintamente, ofrecer respuestas en tiempo real sobre sus tareas, incrementar la creatividad, interacción en tiempo real con los profesores o expertos en el tema de la clase y acceso instantáneo a materiales de la clase (8). Un estudio realizado en México indica que el $84 \%$ de alumnos de 12 facultades universitarias distintas (incluyendo 39 alumnos de una Facultad de Medicina) refiere que las redes sociales les permiten realizar trabajos en equipo en cualquier tiempo y espacio, el $63 \%$ considera que mejora la comunicación e interacción con sus compañeros, el $68 \%$ que ahorran tiempo para preparar trabajos y el $42 \%$ opinan que fomentan el trabajo en grupo y colaborativo (9). Además, en México, el 86\% de los estudiantes indicaron que habían participado de alguna experiencia educativa dentro de una red social y que se comunicaban con sus profesores a través de las redes sociales, más frecuentemente para enviar tareas y resolver dudas de la clase (9). En otro estudio hecho en Arabia Saudí se reporta que el 87,7\% de estudiantes de medicina utiliza las redes sociales en su aprendizaje y educación (10).

En el Perú no existen estudios sobre el uso de redes sociales en estudiantes de medicina. El objetivo de este estudio fue describir algunas formas de uso de algunas redes sociales por los estudiantes de pregrado de una facultad de medicina en la ciudad de Lima.

\section{MATERIAL Y MÉTODOS}

Estudio descriptivo transversal, realizado en abril del 2017 en alumnos del quinto año de Medicina de la Facultad de Medicina (FAMED) de la Universidad Peruana Cayetano Heredia (UPCH).

Se diseñó una encuesta para conocer las formas de comunicación en general que usaban todos los alumnos al inicio del curso de Clínica Médica 2 en la sede del Hospital Nacional Arzobispo Loayza (HNAL), además de la edad y género del alumno, mediante cuatro preguntas específicas sobre redes sociales con alternativas para marcar:

¿En qué red(es) social(es) tiene usted alguna cuenta? Si respondían afirmativamente, debían escoger entre 6 redes sociales: Facebook ${ }^{\circledR}$, Instagram $\AA$, Twitter $\AA$, SlideShare $\AA$, Google $+\AA$ y LinkedIn ${ }^{\circledR}$ (Podían marcar más de una).

¿Con qué frecuencia ingresa usted a su(s) red(es) social(es)? Se les dio 4 alternativas por cada red social que tenían cuenta: Más de 1 vez al día, 1 vez al día, más de 1 vez por semana, 1 vez por semana.

¿Cuánto tiempo utiliza diariamente en ver o postear en Facebook? Se les dio 6 alternativas: Más de 2 horas, 1 a 2 horas, 30 a 60 minutos, 15 a 30 minutos, menos de 15 minutos, menos de 1 vez al día.

¿Con qué frecuencia visualiza videos de YouTube? Se les dio 6 alternativas: Más de 1 vez al 
día, 1 vez al día, más de 1 vez por semana, 1 vez por semana, menos de 1 vez por semana.

No se incluyeron otras redes sociales que tienen un enfoque menos académico o son menos populares (Pinterest ${ }^{\circledR}$, Tumblr ${ }^{\circledR}$ ). Tampoco se incluyó en ese momento otras redes sociales como: Whatsapp ${ }^{\circledR}$ (pues se asumió al inicio de la investigación que todos los alumnos tendrían la aplicación en su celular), ni otras redes sociales o aplicaciones de chat menos populares en Perú o cuyo mayor uso sea menos académico (WeChat ${ }^{\circledR}$, Telegram ${ }^{\circledR}$, Line $\AA$, Tumblr $\AA$, SnapChat $\left.{ }^{\circledR}\right)$.

Una vez construido el instrumento, se procedió a su desarrollo electrónico en la plataforma SurveyMonkey® (www.surveymonkey.com). Dicha encuesta fue enviada por medio de un link a través del correo electrónico de la universidad a todos los alumnos que estaban rotando en ese momento en el curso mencionado y se les solicitó que contesten la encuesta de forma voluntaria. En el mismo correo se les solicitó su autorización para utilizar estos datos. Se configuró que todas las preguntas deberían ser completadas. Todas las respuestas obtenidas fueron anónimas.

Los datos fueron exportados desde la plataforma del Survey Monkey ${ }^{\circledR}$ al programa Excel ${ }^{\circledR} 2013$. Para la estadística descriptiva se usaron frecuencias y porcentajes para las variables categóricas. Para la variable edad se calcularon el promedio, la desviación estándar y los rangos.

El estudio no fue revisado previamente por un comité de ética. Sin embargo, se solicitó el consentimiento para participar en el estudio al enviar la encuesta por mail y se protegió la privacidad y la confidencialidad de los datos, ya que los datos recabados fueron extraídos a un documento u hoja de MS Excel, sin incluir datos que puedan identificar a los participantes. Solo se extrajo de cada encuesta la información presentada en los resultados del estudio. Nadie más sino el propio investigador tuvo acceso a la información de las encuestas.

\section{RESULTADOS}

Se envió la encuesta a 68 alumnos, respondieron 52 (tasa de respuesta 76,4\%). De los alumnos que contestaron, $28(51,9 \%)$ fueron mujeres. El promedio de edad fue 22,1 $\pm 1,6$ años (rango: 20-28 años).

Todos los alumnos que respondieron la encuesta manifestaron tener una cuenta en Facebook $\mathbb{R}$; y en menor proporción, en Google+ $(\AA$, Instagram $\AA$, Twitter ${ }^{\circledR}$, SlideShare ${ }^{\circledR}$ y LinkedIn ${ }^{\circledR}$ (tabla 1). El $1,9 \%$ de alumnos tenían las 5 redes sociales, 34,6\% tenían $3,32,7 \%$ tenían 2 , y $30,8 \%$ tenían solo una red social.

Tabla 1. Número de alumnos que tenían alguna red social.

\begin{tabular}{lcc}
\hline & $\mathbf{n}$ & $\mathbf{\%} *$ \\
\hline Facebook & 52 & 100,0 \\
Google + & 28 & 53,8 \\
Instagram & 24 & 46,2 \\
Twitter & 18 & 34,6 \\
SlideShare & 8 & 15,4 \\
LinkedIn & 4 & 7,7 \\
\hline
\end{tabular}

(*) Algunos alumnos tenían más de una red social

Tabla 2. Frecuencia de uso de cada red social.

\begin{tabular}{lcccccccc}
\hline & $\begin{array}{c}\text { Más de } \mathbf{1} \text { vez al } \\
\text { día }\end{array}$ & \multicolumn{1}{c}{$\mathbf{1}$ vez al día } & \multicolumn{2}{c}{$\begin{array}{c}\text { Más de 1 vez } \\
\text { por semana }\end{array}$} & \multicolumn{2}{c}{$\begin{array}{c}\mathbf{1} \text { vez por } \\
\text { semana }\end{array}$} \\
\cline { 2 - 10 } & $\mathbf{n}$ & $\mathbf{\%}$ & $\mathbf{n}$ & $\mathbf{\%}$ & $\mathbf{n}$ & $\mathbf{\%}$ & $\mathbf{n}$ & $\mathbf{\%}$ \\
\hline Facebook & 41 & 78,8 & 4 & 7,7 & 7 & 13,5 & 0 & 0,0 \\
Google + & 13 & 46,4 & 4 & 14,3 & 7 & 25,0 & 4 & 14,3 \\
Instagram & 10 & 41,7 & 5 & 20,8 & 5 & 20,8 & 4 & 16,7 \\
Twitter & 5 & 27,8 & 1 & 5,6 & 3 & 16,7 & 9 & 50,0 \\
SlideShare & 1 & 12,5 & 0 & 0,0 & 2 & 25,0 & 5 & 62,5 \\
LinkedIn & 0 & 0,0 & 1 & 25,0 & 0 & 0,0 & 3 & 75,0 \\
\hline
\end{tabular}


El $86,5 \%$ y $62,5 \%$ utilizaban Facebook ${ }^{\circledR}$ e Instagram ${ }^{\circledR}$ todos los días, respectivamente. Las otras redes sociales fueron usadas en menor frecuencia (tabla 2).

La frecuencia de acceso a Facebook ${ }^{\circledR}$ durante el día fue de una a más horas en $26(50 \%)$; menos de una hora en 19 (36,5\%); y menos de una vez al día en 7 $(13,5 \%)$ alumnos.

Todos los alumnos respondieron que visualizaban videos en YouTube ${ }^{\circledR}$, con un tiempo invertido de visualización de una o más veces al día en $33(63,5 \%)$; una o más veces por semana en $17(32,7 \%)$; y menos de una vez por semana en $2(3,8 \%)$ alumnos.

\section{DISCUSIÓN}

En la presente investigación, todos los alumnos que respondieron la encuesta tenían al menos una cuenta en Facebook ${ }^{\circledR}$, al igual que un estudio similar en Canadá, realizado en estudiantes de Medicina de la Universidad de Otawa (7).

Ni en nuestro estudio ni en el de Canadá se especificó el uso de Facebook solo en actividades académicas, de distracción o ambas. Sin embargo, en el estudio realizado en Arabia Saudita mencionado anteriormente se encontró que $65,9 \%$ de alumnos usaba Facebook ${ }^{\circledR}$, aunque se preguntó por el uso sólo en su educación universitaria (10). En Australia, solo el $87 \%$ de estudiantes universitarios de primer año de medicina tenían Facebook ${ }^{\circledR}$ (11), y en México, el 64\% de alumnos universitarios de 18 a 35 años de edad reportaron que la red social más utilizada por ellos era Facebook ${ }^{\circledR}(9)$.

A pesar que la mayoría de alumnos universitarios utilizan el Facebook ${ }^{\circledR}$ para actividades sociales, recreativas o de ocio (revisar o comentar una noticia o fotos, jugar online), en un estudio en Lima, Perú, hasta $40 \%$ de ellos lo utilizan para coordinar trabajos o temas de la universidad (10).

En el estudio canadiense (7), el 67\% de los alumnos se conectaban a diario a Facebook $\AA$, a diferencia del $86,5 \%$ de nuestros alumnos, de los cuales el $50 \%$ pasan una hora o más por día conectados a esta red social, porcentaje similar a los alumnos de la Pontificia Universidad Católica del Perú (PUCP) en Lima, Perú, (12).
En Arabia Saudi, 93,8\% de estudiantes de medicina usan YouTube ${ }^{\circledR}$ en su educación (10); en nuestro estudio se encontró un porcentaje menor, a pesar que algunos estudios reportan alguna utilidad y beneficio de YouTube ${ }^{\circledR}$ en la enseñanza-aprendizaje de varios tópicos de la salud y medicina, incluyendo el aprendizaje de farmacocinética $(63 \%$ de videos educacionalmente útiles) (13) y del examen físico del sistema nervioso (47\% de videos útiles) (14).

Aunque algunos autores han reportado que casi $80 \%$ de estudiantes universitarios de la carrera de medicina en Australia completaron tareas de evaluación mediante Twitter ${ }^{\circledR}$ (12), en nuestro estudio esta plataforma fue utilizada por pocos alumnos.

Es importante recalcar, que todo el tiempo invertido diariamente por los alumnos en las redes sociales que se ha evidenciado en nuestro estudio, podría ir en detrimento del desarrollo académico de los alumnos, no solo por distracción sino por tener menos tiempo disponible para la lectura académica. Como ejemplo de esto, $40 \%$ de alumnos en Arabia Saudi (10) refieren que usar las redes sociales puede ser distractor más que educativo, al igual que en México donde $42 \%$ de alumnos refieren que las redes sociales los distraen para realizar sus trabajos universitarios (9). Se requieren otras investigaciones en nuestro medio para comprobar o no dicha consecuencia.

Por otro lado, en el estudio canadiense (7), se evidencia un menor uso de otras redes sociales (Twitter $\AA$, Instagram ${ }^{\circledR}$, Google $+\AA$ ) de forma similar que en nuestros alumnos. Pero, el uso de LinkedIn ${ }^{\circledR}$ fue mayor, ya que el $21 \%$ de alumnos canadienses tenían cuenta comparado con menos de $8 \%$ en nuestro estudio. Como se menciona en la web de LinkedIn ${ }^{\circledR}$, su misión es "conectar a los profesionales del mundo para ayudarles a aumentar su productividad $y$ rendimiento. Al unirse... obtienen acceso a... información que te ayudará a destacar en tu campo profesional" (15). Si bien esta red social es usada más por personas que ya tienen una profesión lograda, y muchas personas la utilizan más para conseguir trabajo, los temas posteados en dicha red social son más de tipo académico y científico y tiene menos distractores que otras redes sociales. Por ello, podría ser de utilidad también a los alumnos universitarios no solo en su educación sino porque en algún momento al terminar la universidad descubrirán su utilidad.

Lo novedoso de nuestra investigación, a diferencia de otros estudios sobre el tema, es que se 
incluyó SlideShare ${ }^{\circledR}$, una web que tiene un enfoque más académico y profesional. No obstante, pocos alumnos (menos de $8 \%$ ) tienen cuenta en esta red social. Por ello, no se evaluó si ellos podían acceder a algún contenido de dicha página web aún sin tener cuenta propia. Desde que SlideShare ${ }^{\circledR}$ se unió a LinkedIn ${ }^{\circledR}$ en el 2012, se convirtió en el destino principal de contenido profesional y académico, haciendo mucho más fácil compartir conocimiento. En su web recomiendan usarla para aprender de manera más rápida, más inteligente y prácticamente cualquier tópico (16). Si bien no es una red social tan popular como otras, la web no solo permite compartir presentaciones, documentos portables (pdf) o de texto, o algunos archivos de audio o video, sino que también permite recibir y dar comentarios a las presentaciones que uno haya publicado o accedido.

Nuestros estudiantes ya utilizan plataformas de redes sociales, entonces como educadores deberíamos reformular el currículo y comprometer a los estudiantes "en sus propios términos" (12). Al incorporar la tecnología digital, los estudiantes pueden asumir más responsabilidad en su propio aprendizaje, acceder a una gran cantidad de información y aprender en cualquier lugar a su propio ritmo, así como compartir conocimientos y experiencias, comunicarse con sus compañeros y profesores y aumentar sus experiencias de aprendizaje (7).

Aunque la tasa de respuesta de nuestra encuesta fue más del $75 \%$, mayor a encuestas realizadas en otros estudios (7), una limitación del estudio puede ser el poco número de alumnos encuestados y quizás una mayor diferenciación en los datos se podría haber obtenido con un número mayor de alumnos. Otra limitación es que no se especificó la razón del uso de las redes sociales o de la visualización de videos hacia el tema académico o recreacional. Otra limitación del estudio es que en ninguna de las preguntas realizadas se especificó el uso de las redes sociales para la educación o el aprendizaje, pues el objetivo de la encuesta no estuvo dirigido a la indagación del uso de las redes sociales para educación, sino para indagar el uso de redes sociales para comunicación general entre alumnos y con su coordinador o profesores durante el curso que estaban llevando dichos alumnos en ese momento. Por último, la respuesta de los alumnos es de modo general, aunque da alcances sobre qué redes sociales se pueden aprovechar para la comunicación con los alumnos, y poder sugerirles la utilización de otras redes sociales más académicas.
En conclusión, se encontró que todos los estudiantes tienen al menos una cuenta en redes sociales, de preferencia en Facebook ${ }^{\circledR}$, lo usan al menos una vez al día, similar a lo encontrado en otros países.

Este es el primer reporte en el Perú del uso de redes sociales para la comunicación entre los estudiantes universitarios de pregrado de la carrera de medicina y que brinda información relevante para utilizar y guiar futuros estudios, ya más dirigidos al ámbito de la educación médica.

Por ello, también se debería investigar sobre el uso de las redes sociales en los profesores, y su utilización con sus alumnos en el proceso de enseñanza aprendizaje en la universidad, porque si la mayoría de profesores de cada universidad no utilizan las redes sociales de una manera frecuente, entonces sería infructuoso dirigir esta tecnología a futuro hacia la docencia, al menos en la actualidad.

\section{Declaración de fuentes de financiamiento y de conflictos de interés:}

El trabajo fue financiado por el autor. Declara no tener algún conflicto de intereses.

\section{Agradecimientos}

A Diana Elizabeth Calle Jacinto, Magister en Gestión del Cuidado de Enfermería en la Universidad Peruana Cayetano Heredia (UPCH), por la revisión del manuscrito.

\section{Correspondencia:}

Guillén López, Otto Barnaby

Avenida Antonio Miroquesada 572 - departamento 1802. Lima 17, Perú.

Teléfono: 51-997851413

Correo electrónico: otto.guillen.1@upch.pe

\section{REFERENCIAS BIBLIOGRÁFICAS}

1. Instituto Nacional de Estadística e Informática. Población que accede a internet. Lima: Instituto Nacional de Estadística e Informática; 2017. (Fecha de acceso: 13 de enero de 2018) Disponible en: https://www.inei.gob.pe/estadisticas/indicetematico/population-access-to-internet/

2. Espiritusanto O. Generación Z: Móviles, redes y contenido generado por el usuario. Revista de Estudios de Juventud. 2017; 114:111-126. (Citado el 19 de 
enero de 2018) Disponible en: http://www. injuve.es/sites/default/files/2017/28/publicaciones/ documentos_8._generacion_._moviles_redes_y contenido_generado_por_el_usuario.pdf

3. Real Academia Española. Red. Madrid: Diccionario de la lengua española; 2018. (Citado el 08 de enero de 2019). Disponible en: http://dle.rae.es/?id=VXs6SD8

4. Cambridge Dictionary. Social network. Cambridge: Cambridge University Press; 2019. (Citado el 08 de enero de 2019). Disponible en: ttps://dictionary. cambridge.org/es/diccionario/ingles/social-network

5. Computer Hope. Social network. Utah: Computer Hope; 2017. (Citado el 08 de enero de 2019). Disponible en: https://www.computerhope.com/ jargon $/ \mathrm{s} /$ socinetw.htm

6. Institute of Education of University College London. Social Media for Researchers: Slideshare. London: Institute of Education of University College London; 2018. (Citado el 08 de enero de 2019). Disponible en: http://libguides.ioe.ac.uk/c.php?g=482417\&p=3299 150

7. El Bialy, S, Jalali A. Go where the students are: A comparison of the use of social networking sites between medical students and medical educators. JMIR Med Educ 2015; 1(2):e7. doi:10.2196/ mededu. 4908

8. George DR, Dellasega C. Use of social media in graduate level medical humanities education: Two pilot studies from Penn State College of Medicine. Med Teach. 2011; 33(8):e429-34. doi: 10.3109/0142159X.2011.586749

9. López-González R, López-Flores MP. Uso de las redes sociales digitales en el ámbito escolar universitario. Estud $\lambda$ ambda teór pract didact leng lit. 2017; 2: 25-45. (Citado el 18 de octubre de 2017) Disponible en: http://estudioslambda.uson.mx/ revistas/articulos/19-16-2-r2_art02.pdf
10. Alsuraihi AK, Almaqati AS, Abughanim SA, Jastaniah NA. Use of social media in education among medical students in Saudi Arabia. Korean J Med Educ. 2016; 28(4): 343-354. Doi: https://doi. org/10.3946/kjme.2016.40

11. Diug B, Kendal E, Ilic D. Evaluating the use of twitter as a tool to increase engagement in medical education. Educ Health. 2016; 29:223-30.

12. Deza MI. Uso y soporte social percibido en Facebook en una muestra de estudiantes universitarios. Tesis de Licenciatura en Psicología. Lima, Perú: Pontificia Universidad Católica del Perú; 2014. 71 pp. (Citado el30deoctubrede2017)Disponibleen:http://tesis.pucp. edu.pe/repositorio/bitstream/handle/123456789/ 5878/DEZA_DE_LA_VEGA_MARIA_USO_ FACEBOOK.pdf? sequence $=3 \&$ isAllowed $=y$

13. Azer SA. Understanding pharmacokinetics: are YouTube videos a useful learning resource? Eur Rev Med Pharmacol Sci. 2014; 18: 1957-1967.

14. Azer SA, AlEshaiwi SM, AlGrain HA, AlKhelaif RA. Nervous system examination on YouTube. BMC Med Educ.2012; 12:126.

15. LinkedIn. Sobre nosotros. California: LinkedIn Corporation; 2017. (Citado el 19 de enero de 2018) Disponible en: https://press.linkedin.com/es-es/ about-linkedin

16. LinkedIn SlideShare. About. California: LinkedIn Corporation; 2017. (Citado el 19 de enero de 2018) Disponible en: https://es.slideshare.net/linkedin

Recibido: 13/09/2018

Aceptado: 28/03/2019 